\title{
Ventilação Mecânica no Intra-Operatório
}

\author{
Intraoperative Mechanical Ventilation
}

\author{
José Otávio Costa Auler Junior ${ }^{1}$, Filomena Regina Barbosa Gomes Galas²,
} Ludhmila Abrahão Hajjar ${ }^{3}$, Suelene Aires Franca ${ }^{4}$

\section{RESUMO}

JUSTIFICATIVA E OBJETIVOS: Em 2000, foi publicado o II Consenso Brasileiro de Ventilação Mecânica. Desde então, o conhecimento na área da ventilação mecânica avançou rapidamente, com a publicação de inúmeros estudos clínicos que acrescentaram informações importantes para o manuseio de pacientes críticos em ventilação artificial. Além disso, a expansão do conceito de Medicina Baseada em Evidências determinou a hierarquização das recomendações clínicas, segundo o rigor do método dos estudos que os embasaram. Essa abordagem explícita vem ampliando a compreensão e a aplicação das recomendações clínicas. Por esses motivos, a AMIB - Associação de Medicina Intensiva Brasileira - e a SBPT - Sociedade Brasileira de Pneumologia e Tisiologia - julgaram conveniente a atualização das recomendações descritas no Consenso anterior. Dentre os tópicos selecionados a Ventilação Mecânica no Intra-Operatório foi um dos temas propostos. O objetivo foi descrever os pontos mais importantes relacionados à ventilação mecânica no período intra-operatório.

MÉTODO: Objetivou-se chegar a um documento suficientemente sintético, que refletisse a melhor evidência disponível na literatura. A revisão bibliográfica baseouse na busca de estudos através de palavras-chave e em sua gradação conforme níveis de evidência. As palavras-chave utilizadas para a busca foram: mechanical ventilation, perioperative e anesthesia.

RESULTADOS: São apresentadas recomendações quanto à prevenção de complicações, as modalidades ventilatórias que podem ser aplicadas durante a anes-

1. Professor Titular de Anestesiologia da Faculdade de Medicina da Universidade de São Paulo (SP)

2. UTI da Divisão de Anestesiologia do Instituto do Coração da Faculdade de Medicina da Universidade de São Paulo (SP)

3. UTI da Divisão de Anestesiologia do Instituto do Coração da Faculdade de Medicina da Universidade de São Paulo (SP)

4. UTI do Hospital das Clínicas da Universidade Federal de Goiás (GO)

(C)Associação de Medicina Intensiva Brasileira, 2007 tesia e as manobras pós-operatórias para aumento do volume pulmonar.

CONCLUSÕES: A atelectasia pulmonar tem se mostrado uma complicação freqüente no intra-operatório, assim técnicas de ventilação mecânica têm sido introduzidas visando a redução nessas complicações.

Unitermos: Anestesia, Complicações, Intra-Operatório, Ventilação Mecânica

\section{SUMMARY}

BACKGROUND AND OBJECTIVES: The II Brazilian Consensus Conference on Mechanical Ventilation was published in 2000. Knowledge on the field of mechanical ventilation evolved rapidly since then, with the publication of numerous clinical studies with potential impact on the ventilatory management of critically ill patients. Moreover, the evolving concept of evidence - based Medicine determined the grading of clinical recommendations according to the methodological value of the studies on which they are based. This explicit approach has broadened the understanding and adoption of clinical recommendations. For these reasons, AMIB - Associação de Medicina Intensiva Brasileira and SBPT - Sociedade Brasileira de Pneumologia e Tisiologia - decided to update the recommendations of the II Brazilian Consensus. Intraoperative mechanical ventilation has been one of the updated topics. This objective was described the most important topics on the intraoperative mechanical ventilation.

METHODS: Systematic review of the published literature and gradation of the studies in levels of evidence, using the key words: mechanical ventilation, intraoperative and anesthesia.

RESULTS: Recommendations on how to prevent pulmonary complications during anesthesia/postoperative period and which are the best intraoperative ventilatory techniques.

CONCLUSIONS: Pulmonary atelectasis is a common complication observed in the postoperative period, thus ventilatory techniques has been adapted to pre- 
vent these complications.

Key Words: Anesthesia, Intraoperative, Mechanical ventilation, Pulmonary complications.

\section{INTRODUÇÃO}

Em 2000, foi publicado o II Consenso Brasileiro de Ventilação Mecânica. Desde então, o conhecimento na área da ventilação mecânica avançou rapidamente, com a publicação de inúmeros estudos clínicos que acrescentaram informações importantes para o manuseio de pacientes críticos em ventilação artificial. Além disso, a expansão do conceito de Medicina Baseada em Evidências determinou a hierarquização das recomendações clínicas, segundo o rigor do método dos estudos que os embasaram. Essa abordagem explícita vem ampliando a compreensão e a aplicação das recomendações clínicas. Por esses motivos, a AMIB - Associação de Medicina Intensiva Brasileira - e a SBPT - Sociedade Brasileira de Pneumologia e Tisiologia - julgaram conveniente a atualização das recomendações descritas no Consenso anterior. Dentre os tópicos selecionados a Ventilação Mecânica no Intra-Operatório foi um dos temas propostos.

Quadro 1 - Graus de Recomendação

\begin{tabular}{ll}
\hline $\begin{array}{l}\text { Graus de Re- } \\
\text { comendação }\end{array}$ & Tratamento/Prevenção \\
\hline A & Revisão sistemática (com homogeneidade) de \\
& ensaios clínicos controlados e aleatórios; \\
& Ensaio clínico controlado e aleatório com Inter- \\
& valo de confiança estreito; \\
& Resultados terapêuticos do tipo "tudo ou nada". \\
& Revisão sistemática (com homogeneidade) de \\
B & estudos de coorte; \\
& Estudo de coorte (incluindo ensaio clínico alea- \\
& tório de menor qualidade); \\
& Observação de resultados terapêuticos (outco- \\
& mes research) / estudo ecológico; \\
& Revisão sistemática (com homogeneidade) de \\
& estudos caso-controle; \\
& Estudo caso-controle. \\
& Relato de casos (incluindo coorte ou caso-con- \\
& trole de menor qualidade). \\
Opinião de especialista sem avaliação crítica ou \\
baseada em matérias básicas (estudo fisiológi- \\
co ou estudo com animais).
\end{tabular}

\section{DEFINIÇÃO E EPIDEMIOLOGIA}

As complicações pulmonares peri-operatórias são causas significativas de morbimortalidade. A determinação da freqüência e impacto clínico dessas complicações é comprometida pela falta de uma definição uniforme de complicação pulmonar peri-operatória entre os estudos. A maioria dos investigadores inclui nessa definição pneumonia (confirmada ou suspeita), insuficiência respiratória (usualmente definida como a necessidade de ventilação mecânica), broncoespasmo, atelectasias e hipoxemia ${ }^{1}$. Apesar dessa variabilidade nas definições, é ainda evidente que essas complicações ocorrem com freqüência.

Em estudo de pacientes submetidos à cirurgia abdominal eletiva, complicações pulmonares ocorreram mais freqüentemente que complicações cardíacas $(10 \%$ e $6 \%$, respectivamente) e foi associada com maior morbidade $^{2}$.

O manuseio peri-operatório deve contemplar medidas profiláticas e estratégias de proteção das vias aéreas que visem à redução da incidência e do impacto dessas complicações na evolução pós-operatória dos pacientes.

A etiologia das complicações pulmonares pós-operatórias é complexa e pouco compreendida. Tanto as condições clínicas dos pacientes quanto os efeitos sinérgicos da anestesia geral e do procedimento cirúrgico na homeostase do sistema respiratório são responsáveis pela ocorrência dessas complicações.

Para prevenir e reduzir a incidência das complicações pós-operatórias, deve-se avaliar cuidadosamente as condições clínicas dos pacientes e promover intervenções capazes de reduzir o impacto delas na ocorrência das complicações pulmonares. Estas acontecem mais comumente nos pacientes submetidos à anestesia geral, especialmente na cirurgia torácica ou de abdômen superior. Outros fatores de risco são tabagismo, doença pulmonar crônica preexistente, cirurgia de emergência, tempo de anestesia maior ou igual a 180 min e idade avançada.

\section{FISIOPATOLOGIA}

A anestesia geral pode determinar inúmeros efeitos biológicos no sistema respiratório e contribuir para o aparecimento de complicações pulmonares peri-operatórias. Durante a anestesia geral, ocorre diminuição do número e da atividade dos macrófagos, inibição da depuração mucociliar, aumento da permeabilidade alvéolo-capilar, inibição da liberação de surfactante, aumento da atividade da enzima óxido nítrico sintase e acentuação da sensibilidade da vasculatura pulmonar aos mediadores neurohumorais. Após sua indução, ocorre diminuição da capacidade residual funcional 
(CRF), formação de atelectasias nas porções dependentes dos pulmões e alterações significativas dos movimentos do diafragma. O desequilíbrio ventilação/ perfusão resulta em efeito shunt e aumento do espaço morto. Essas alterações fisiopatológicas na CRF, na movimentação diafragmática e na relação ventilação/ perfusão resultam no aumento do gradiente alvéolo-arterial de oxigênio, o que, em parte, é responsável pela necessidade de suplementação de oxigênio no perioperatório.

A atelectasia pulmonar é a principal causa de hipoxemia pós-operatória, ocorrendo em quase $90 \%$ dos pacientes que são submetidos à anestesia, e desempenha papel fundamental nas alterações das trocas gasosas e na redução da complacência estática associada com a lesão pulmonar. Os principais fatores associados à ocorrência das atelectasias são: a compressão do parênquima pulmonar, a absorção do ar alveolar e o comprometimento da função do surfactante.

Assim como a anestesia, o trauma cirúrgico pode resultar na redução da CRF e da capacidade vital (CV) e em atelectasias pulmonares. Estão também envolvidos os mecanismos inflamatórios do trauma cirúrgico, a estimulação reflexa durante a instrumentação da via aérea e a alteração do movimento mucociliar.

Além disso, algumas complicações respiratórias são relacionadas especificamente com determinadas intervenções anestésicas ou cirúrgicas, tais como lesão pulmonar aguda (LPA) após circulação extracorpórea, pneumotórax causado por barotrauma ou trauma cirúrgico, edema pulmonar por pressão negativa após obstrução de vias aéreas durante ventilação espontânea e pneumonia aspirativa ${ }^{3,4}$.

A seguir estão pontuadas as recomendações no manuseio peri-operatório que buscam a redução da ocorrência das complicações pulmonares.

\section{PREVENÇÃO DAS COMPLICAÇÕES PERI-OPERA- TÓRIAS}

\section{Medicações Pré-Operatórias}

Recomendação: Não há justificativa suficiente para recomendar o emprego de medicações ( $\beta$-agonistas, aminofilina, corticosteróides ou antibióticos) com o objetivo de diminuir as complicações pulmonares.

\section{Grau de Recomendação: B}

Comentário: Embora esses esquemas terapêuticos não devam ser utilizados de maneira rotineira em todos os pacientes, a função pulmonar pré-operatória deve ser otimizada de acordo com as necessidades in- dividuais de cada paciente. Por exemplo, os sintomas de asma devem estar controlados (tratamento tópico ou sistêmico), visto que pacientes com sintomas mais ativos apresentam maior freqüência de complicações pulmonares peri-operatórias ${ }^{5,6}$.

\section{Cessação do Tabagismo}

Recomendação: Todos os pacientes devem ser encorajados a cessar o tabagismo, um significativo fator de risco para complicações pulmonares peri-operatórias. O momento ideal da cessação não é conhecido e deve ser foco de futuras investigações.

\section{Grau de Recomendação: B}

Comentário: Alguns estudos demonstram que a redução ou cessação recente do tabagismo (dentro de aproximadamente dois meses antes da cirurgia) pode, paradoxalmente, aumentar o risco de complicações pulmonares peri-operatórias ${ }^{7,8}$. Entretanto, esses estudos não são conclusivos, devido a viés de seleção, já que são os pacientes mais graves aqueles que mais provavelmente reduzem ou cessam o hábito de fumar.

\section{Anestesia Locorregional}

Recomendação: A opção pela anestesia locorregional deve ser feita, quando possível, em pacientes com doenças pulmonares prévias, pois essa modalidade associase à menor incidência de complicações pulmonares, por diversos mecanismos, destacando-se a prevenção da irritação reflexa da manipulação das vias aéreas.

\section{Grau de Recomendação: C}

Comentário: Os resultados de muitos estudos não demonstram vantagem clara de uma técnica anestésica em relação à outra, apesar de, na anestesia geral, haver manipulação das vias aéreas, alteração do controle ventilatório e, em muitos casos, a utilização de bloqueadores neuromusculares, com seu potencial de indução de hipersecreção e broncoespasmo ${ }^{4,9}$.

\section{Ventilação Mecânica}

A troca gasosa pulmonar é sistematicamente comprometida durante a anestesia geral com ventilação mecânica, resultando em reduzida oxigenação arterial. A principal causa é o colapso do tecido pulmonar (atelectasia), presente em quase $90 \%$ dos pacientes anestesiados ${ }^{10,11}$. Já foi demonstrada boa correlação entre a quantidade de atelectasia e o shunt pulmonar $^{10}$, sendo uma preocupação do anestesiologista o conhecimento e a utilização de procedimentos que visem a prevenção da formação de atelectasias e/ou a reabertura de áreas pulmonares colapsadas. 


\section{Pressão Controlada versus Volume Controlado}

Recomendação: Não se recomenda a opção por uma modalidade ventilatória em detrimento da outra com o objetivo de prevenir complicações pulmonares.

\section{Grau de Recomendação: C}

Comentário: A comparação das diferentes modalidades ventilatórias no intra-operatório não demonstrou benefício de uma técnica em relação à outra. Em pacientes submetidos à cirurgia cardíaca, na qual o emprego de ventilação com pressão controlada é bastante utilizado, essa técnica associou-se a aumento significativo do índice cardíaco, diminuição da resistência vascular sistêmica e da pressão de platô, quando comparada com a ventilação controlada a volume. Entretanto, em recente estudo foi demonstrado efeito semelhante das técnicas no que se refere à melhora da hipoxemia pós-operatória' ${ }^{12}$.

\section{Volume-Corrente}

Recomendação: Recomenda-se a utilização de volume-corrente de 8 a $10 \mathrm{~mL} / \mathrm{kg}$ na modalidade volume controlado ou pico/platô de pressão inspiratória suficiente para manter este mesmo volume na modalidade pressão controlada.

\section{Grau de Recomendação: C}

Comentário: A utilização de volume-corrente variável não é prática muito utilizada durante ventilação mecânica em pacientes anestesiados. Nesse período, ocorrem várias alterações da mecânica pulmonar, relacionadas principalmente ao tipo de cirurgia, presença de afastadores, compressão extrínseca e utilização de bloqueadores neuromusculares. Entretanto, sabe-se que o emprego de elevados volumes-correntes pode estar associado com a ocorrência de altas pressões alveolares, hiperdistensão pulmonar e liberação de mediadores inflamatórios que determinam importantes alterações da função pulmonar. Apesar da escassez de estudos que comparem em anestesia a estratégia de baixo volume-corrente versus alto volume-corrente, transpõe-se para a prática peri-operatória os resultados nos trabalhos em lesão pulmonar aguda (LPA) / síndrome da angústia respiratória aguda (SARA), recomendando-se a não utilização de altos volumes-correntes, para se evitar a hiperdistensão alveolar ${ }^{3,6}$.

\section{Pressão Positiva ao Final da Expiração (PEEP)}

Recomendação: A aplicação da PEEP durante anestesia geral é recomendada por associar-se à melhora da oxigenação e prevenção da formação de atelectasias.

\section{Grau de Recomendação: B}

Comentário: Estudos recentes demonstram que a aplicação da PEEP mínima de $5 \mathrm{cmH}_{2} \mathrm{O}$ no intra-operatório resulta em melhora dos parâmetros de oxigenação no intra e no pós-operatório, com redução na formação de atelectasias. Os estudos atuais sugerem aplicação da PEEP em todos os pacientes submetidos à anestesia geral, especialmente nos pacientes de maior risco de complicações pulmonares ${ }^{13,14}$.

\section{Manobras de Recrutamento Alveolar}

Recomendação: A utilização das manobras de recrutamento é prática recomendada no intra-operatório com o objetivo de evitar o colapso alveolar.

\section{Grau de Recomendação: B}

Comentário: Imediatamente após a instalação da anestesia geral, ocorre o aparecimento de atelectasias em áreas dependentes, que são responsáveis pelas alterações da oxigenação no período peri-operatório. A utilização das manobras de recrutamento alveolar, associadas ao emprego da PEEP neste período, é fundamental na abertura dos alvéolos colabados e na manutenção de sua patência, resultando em melhora da oxigenação. Estudos recentes demonstraram benefícios das manobras de recrutamento intra-operatório na prevenção de hipoxemia pós-operatória, sem ocasionar prejuízo hemodinâmico.

Um estudo demonstrou que em cirurgia cardíaca a não utilização de PEEP ou sua utilização entre 5 e 10 $\mathrm{cmH}_{2} \mathrm{O}$, sem manobras de recrutamento, não resultou em melhora da oxigenação, apesar de melhorar o volume-corrente.

Estudo sobre manobras de recrutamento alveolar em pacientes sob anestesia geral e em ventilação mecânica, foi necessária uma pressão de insuflação de $40 \mathrm{cmH}_{2} \mathrm{O}$, mantida por 15 segundos, para se obter completa reabertura de todo o tecido pulmonar colapsado ${ }^{15}$. Devido a preocupações quanto a eventos cardiovasculares adversos decorrentes da estratégia utilizada, o mesmo grupo publicou, posteriormente, um estudo mostrando que, em adultos com pulmões normais, a utilização de pressão de insuflação de 40 $\mathrm{cmH}_{2} \mathrm{O}$ mantida por não mais que 7 a 8 segundos é capaz de re-expandir todo o tecido pulmonar previamente colabado ${ }^{16}$.

Uma outra técnica que tem sido utilizada para recrutamento é a aplicação de CPAP (pressão positiva contínua nas vias aéreas) de 20,30 ou $40 \mathrm{cmH}_{2} \mathrm{O}$ por 20 a 30s. Apesar de existirem estudos com bons resultados em termos de segurança e reversão de hipoxemia, 
com este método no ambiente da Terapia Intensiva ${ }^{17}$, faltam estudos específicos na sala cirúrgica'.

\section{Fração Inspirada de Oxigênio}

Recomendação: Na indução anestésica recomendase a utilização de fração inspirada de $\mathrm{O}_{2}$ de 1 , para assegurar oxigenação adequada para a realização da intubação. Esta prática tem sido associada à formação de atelectasias. Recomendam-se frações de oxigênio necessárias para manutenção da $\mathrm{SpO}_{2}$ acima de $98 \%$. $\mathrm{Na}$ manutenção da anestesia, deve-se utilizar fração inspirada de oxigênio suficiente para manter a saturação de oxigênio acima de $98 \%$.

\section{Grau de Recomendação: C}

Comentário: A utilização de baixas frações inspiradas de oxigênio (abaixo de 0,4$)$ não é recomendada durante a indução anestésica, por reduzir a margem de segurança, caso haja dificuldade de manipulação da via aérea. A utilização da PEEP durante a indução pode prevenir as atelectasias potencializadas pelas altas taxas de oxigênio e deve ser sempre considerada. Em estudo com pacientes adultos normais, a utilização de $10 \mathrm{cmH}_{2} \mathrm{O}$ de PEEP reduziu significativamente a formação de atelectasias, mesmo quando altas frações inspiradas de oxigênio foram usadas ${ }^{18}$. A limitação do uso de altas frações de oxigênio somente à fase de indução anestésica promoverá a prevenção da formação de atelectasias durante a subseqüente fase de manutenção da anestesia ${ }^{19}$. Assim, a ventilação durante a manutenção da anestesia deve ser feita com moderada fração de oxigênio inspirado $\left(\mathrm{FIO}_{2}\right.$ em torno de 0,3 a 0,4$)$, que deve ser aumentada somente em caso de comprometimento da oxigenação arterial ${ }^{14,20}$.

\section{Desmame da Ventilação Mecânica}

Recomendação: O desmame da ventilação mecânica (VM) pode ser realizado utilizando-se pressão de suporte (PSV) ou ventilação mandatória intermitente sincronizada (SIMV).

\section{Grau de Recomendação: C}

Comentário: O desmame da ventilação mecânica no pós-operatório caracteriza-se por aumento de estresse cardiovascular e metabólico. Sendo assim, deve-se progredir o desmame quando o paciente apresenta-se hemodinamicamente estável, equilibrado do ponto de vista hidroeletrolítico, com analgesia adequada e nível de consciência suficiente para o controle ventilatório. A extubação pode ser realizada na sala cirúrgica, na sala recuperação pós-anestésica ou na unidade de terapia intensiva, desde que os critérios descritos sejam obedecidos ${ }^{3}$.

\section{Analgesia Pós-Operatória}

Recomendação: A obtenção de analgesia pós-operatória adequada associa-se à otimização da função pulmonar pós-operatória.

\section{Grau de Recomendação: B}

Comentário: Recomenda-se analgesia eficaz no pósoperatório como método de redução das complicações pulmonares. Tem sido discutido qual a melhor modalidade de analgesia pós-operatória para a prevenção dessas complicações. Há estudos que demonstram a superioridade da analgesia peridural na profilaxia dessas complicações, embora os dados sejam conflitantes ${ }^{21,23}$.

\section{Manobras Pós-Operatórias para Aumento do Volu- me Pulmonar}

Recomendação: Manobras pós-operatórias para aumentar os volumes pulmonares médios são comprovadamente associadas à redução de complicações pósoperatórias.

\section{Grau de Recomendação: B}

Comentário: Dentre os métodos utilizados para aumento do volume pulmonar pós-operatório, destacam-se a ventilação com pressão positiva intermitente, exercícios de respiração profunda, espirometria de incentivo e fisioterapia respiratória ${ }^{24}$.

\section{REFERÊNCIAS}

01. Warner DO - Preventing postoperative pulmonary complications: the role of the anesthesiologist. Anesthesiology, 2000;92:1467-1472.

02. Lawrence VA, Hilsenbeck SG, Mulrow CD et al - Incidence and hospital stay for cardiac and pulmonary complications after abdominal surgery. $J$ Gen Intern Med, 1995;10:671-678.

03. Rock P, Rich PB - Postoperative pulmonary complications. Curr Opin Anaesthesiol, 2003;16:123-131.

04. Weiss YG, Merin G, Koganov E et al - Postcardiopulmonary bypass hypoxemia: a prospective study on incidence, risk factors, and clinical significance. J Cardiothorac Vasc Anesth, 2000;14:506-513.

05. Warner DO, Warner MA, Barnes RD et al - Perioperative respiratory complications in patients with asthma. Anesthesiology, 1996;85:460-467.

06. Weissman C - Pulmonary complications after cardiac surgery. Semin Cardiothorac Vasc Anesth, 2004;8:185-211.

07. Bluman LG, Mosca L, Newman N et al - Preoperative smoking habits and postoperative pulmonary complications. Chest, 1998;113:883-889.

08. Warner MA, Offord KP, Warner ME et al - Role of preoperative cessation of smoking and other factors in postoperative pulmonary complications: a blinded prospective study of coronary artery bypass patients. Mayo Clin Proc, 1989;64:609-616.

09. Rodgers A, Walker N, Schug S et al - Reduction of postoperative mortality and morbidity with epidural or spinal anaesthesia: results from overview of randomised trials. BMJ, 2000;321(7275):1493.

10. Gunnarsson L, Tokics L, Gustavsson $\mathrm{H}$ et al - Influence of age on atelectasis formation and gas exchange impairment during general anaesthesia. Br J Anaesth, 1991;66:423-432.

11. Strandberg A, Tokics L, Brismar B et al - Atelectasis during anaesthesia and in the postoperative period. Acta Anaesthesiol Scand, 1986;30:154-158.

12. Auler Junior JO, Carmona MJ, Silva MH et al - Haemodynamic effects of pressure-controlled ventilation versus volume-controlled ventilation in patients submitted to cardiac surgery. Clin Intensive Care, 1995;6:100-106. 


\section{AULER JUNIOR, GALAS, HAJJAR E COL.}

13. Gander S, Frascarolo P, Suter M et al - Positive end-expiratory pressure during induction of general anesthesia increases duration of nonhypoxic apnea in morbidly obese patients. Anesth Analg, 2005;100:580-584.

14. Rusca M, Proietti S, Schnyder P et al - Prevention of atelectasis formation during induction of general anesthesia. Anesth Analg, 2003;97:1835-1839.

15. Rothen HU, Sporre B, Engberg G et al - Re-expansion of atelectasis during general anaesthesia: a computed tomography study. $\mathrm{Br} \mathrm{J}$ Anaesth, 1993;71:788-795.

16. Rothen HU, Neumann P, Berglund JE et al - Dynamics of re-expansion of atelectasis during general anaesthesia. Br J Anaesth, 1999;82:551-556.

17. Amato MB, Barbas CS, Medeiros DM et al - Beneficial effects of the "open lung approach" with low distending pressures in acute respiratory distress syndrome. A prospective randomized study on mechanical ventilation. Am J Respir Crit Care Med, 1995;152:1835-1846.

18. Neumann P, Rothen HU, Berglund JE et al - Positive end-expiratory pressure prevents atelectasis during general anaesthesia even in the presence of a high inspired oxygen concentration. Acta Anaesthesiol Scand, 1999;43:295-301.

19. Reber A, Engberg G, Wegenius G et al - Lung aeration. The effect of pre-oxygenation and hyperoxygenation during total intravenous anaesthesia. Anaesthesia, 1996;51:733-737.

20. Rothen HU, Sporre B, Engberg G et al - Influence of gas composition on recurrence of atelectasis after a reexpansion maneuver during general anesthesia. Anesthesiology, 1995;82:832-842.

21. Jayr $\mathrm{C}$, Thomas $\mathrm{H}$, Rey $\mathrm{A}$ et al - Postoperative pulmonary complications. Epidural analgesia using bupivacaine and opioids versus parenteral opioids. Anesthesiology, 1993;78:666-676.

22. Ballantyne JC, Carr DB, deFerranti S et al - The comparative effects of postoperative analgesic therapies on pulmonary outcome: cumulative meta-analyses of randomized, controlled trials. Anesth Analg, 1998;86:598-612.

23. Simonneau G, Vivien A, Sartene R et al - Diaphragm dysfunction induced by upper abdominal surgery. Role of postoperative pain. Am Rev Respir Dis, 1983;128:899-903.

24. Celli BR, Rodriguez KS, Snider GL - A controlled trial of intermittent positive pressure breathing, incentive spirometry, and deep breathing exercises in preventing pulmonary complications after abdominal surgery. Am Rev Respir Dis, 1984;130:12-15. 